\title{
Pentaquark at JLab: the g11 experiment in CLAS
}

\author{
M.Battaglieri*, R. De Vita*, V.Kubarovsky ${ }^{\dagger, * *}$ and the CLAS \\ Collaboration \\ * Istituto Nazionale di Fisica Nucleare - Via Dodecaneso 3316139 Genova ITALY \\ ${ }^{\dagger}$ Rensselaer Polytechnic Institute - Troy New York 12180-359 \\ ${ }^{* *}$ Jefferson Laboratory - 12000 Jefferson Avenue Newport News 23606 Virginia
}

\begin{abstract}
After the claiming of the possible discovery of a pentaquark state, many experiments reported positive and negative results opening a discussion about the pentaquark existence. New experiments with high resolution and high statistics are needed to solve the controversy. Jefferson Lab started a comprehensive program to search for pentaquark in photoproduction at threshold on proton and deuteron targets, collecting more than 10 times the existing statistics. The first experiment on the proton (g11) just finished to analyze the data and in a short time will be able to report about the pentaquark search.
\end{abstract}

Keywords: Pentaquark, photoproduction, proton target.

PACS: $13.60 \mathrm{Rj} ; 25.20 \mathrm{Lj}$

\section{INTRODUCTION}

The possible existence of a narrow resonance with strangeness quantum number $\mathrm{S}=+1$ and valence quark structure $u d u d \bar{s}$ was reported by the LEPS Collaboration [1] and then confirmed by many other experiments using a wide variety of probes (photon, electron, protons) and targets (proton, deuteron, nuclei). This state, known as $\Theta^{+}$was predicted to be few $\mathrm{MeV}$ wide with a mass of about $1540 \mathrm{MeV}$, in very good agreement with the experimental findings. If it exists, this would be the first example of a baryon state that is not made up of a simple 3-quark (qqq) valence configuration. However, the low statistics of the observed structures and many null results obtained from the reanalysis of high energy experiments questioned the pentaquark existence. The experimental evidence, both positive or negative, was obtained from data previously collected for other purposes in many reaction channels and very different kinematic conditions, which may involve different production mechanisms. Thus a direct comparisons of the results of the different experiments are very difficult, preventing a definitive conclusion. A second generation of dedicated experiments, optimized for the pentaquark search, was undertaken at Jefferson Laboratory (JLab). These experiments cover the few $\mathrm{GeV}$ region where most of the positive evidence were reported, and collected at least an order of magnitude more statistics than the previous measurements. The mass resolution is of the order of few $\mathrm{MeV}$ and the accuracy in the mass determination is of $1-2 \mathrm{MeV}$, allowing a precise determination of any possible narrow peaks in the spectra. In this talk we report about the so called g11 experiment run with real photon on a proton target. 

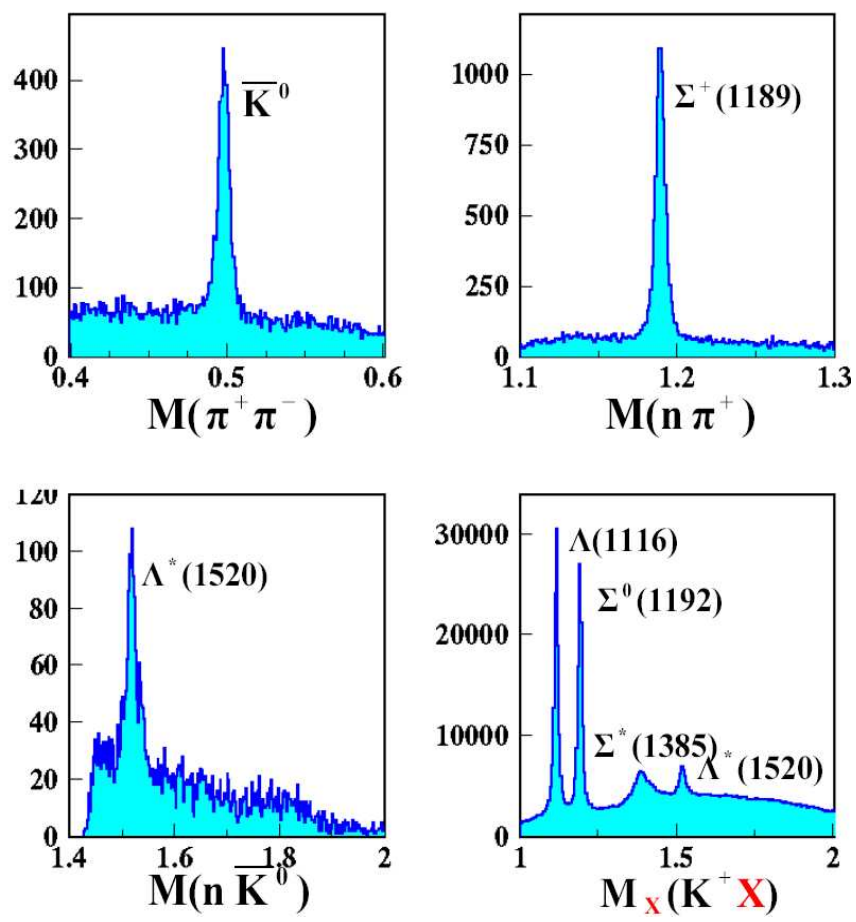

FIGURE 1. Missing and invariant masses for different final states measured in CLAS. Error on mass determination of known baryons and mesons is within 1-2 MeV of the nominal PDG value.

\section{THE G11 EXPERIMENT AT JLAB}

The g11 experiment aimed to measure two production channels on the proton: $\bar{K}^{0} \Theta^{+}$ and $K^{*} \Theta^{+}$, each using two decay modes of the $\Theta^{+}: K^{+} n$ and $K^{0} p$, for a total of four final states. In particular, the reaction $\gamma p \rightarrow \bar{K}^{0} K^{+} n$ was previously investigated at ELSA by the SAPHIR collaboration [2] in a similar photon energy range, finding a positive evidence for a narrow $\Theta^{+}$state with $\mathrm{M}=1540 \mathrm{MeV}, \mathrm{FWHM} \Gamma<25 \mathrm{MeV}$, and a production cross section of the order of $300 \mathrm{nb}$. The observed peak had 55 events in it over a background of 56 events. This result called for a confirmation from an high resolution and high statistics experiment.

The measurement was performed using the CLAS detector in Hall-B with a bremsstrahlung photon beam produced by a continuous $60 \mathrm{nA}$ electron beam of $E_{0}=$ $4.0 \mathrm{GeV}$ impinging on a gold foil $8 \times 10^{-5}$ radiation lengths thick. A bremsstrahlung tagging system with a photon energy resolution of $0.1 \% E_{0}$ was used to tag photons in the energy range from $1.6-3.8 \mathrm{GeV}$. A liquid hydrogen target was contained in a mylar cylinder cell $4 \mathrm{~cm}$ in diameter and $40 \mathrm{~cm}$ long. Outgoing hadrons were detected in the CLAS [3] spectrometer. Momentum information for charged particles was obtained via tracking through three regions of multi-wire drift chambers immersed in a toroidal 


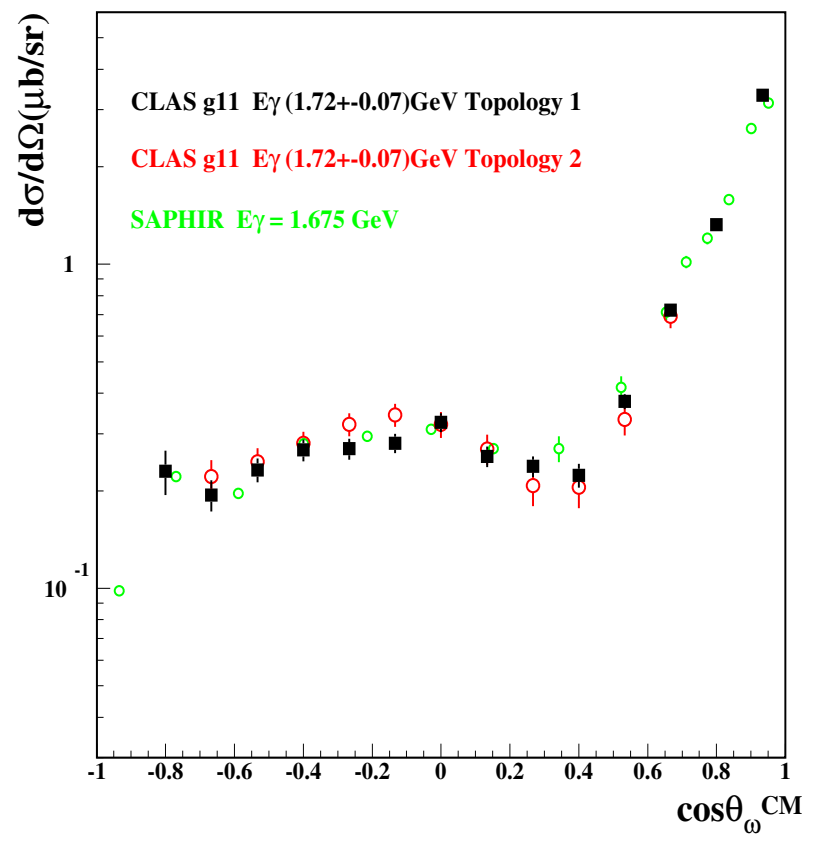

FIGURE 2. The differential cross section obtained by the two topologies and compared to existing data

magnetic field $(\sim 0.5 \mathrm{~T})$, which was generated by six superconducting coils. The field was set to bend the positive particles away from the beam into the acceptance region of the detector. Time-of-flight scintillators (TOF) were used for hadron identification. The interaction time between the incoming photon and the target was measured by the Start Counter (ST), consisting of a set of $24,2.2 \mathrm{~mm}$ thick plastic scintillators surrounding the hydrogen cell. The CLAS momentum resolution is of the order of $0.5-1 \%$ depending on the kinematics. The detector geometrical acceptance for each positive particle in the relevant kinematic region is about $40 \%$. It is somewhat less for low energy negative hadrons, which can be lost at forward angles because they are bent out of the acceptance by the toroidal field. Coincidences between the photon tagger and the CLAS detector triggered the recording of the events. The trigger in CLAS was defined requiring the coincidence between the TOF system and the ST in at least two sectors. We took data for 50 days during June and July 2004 collecting more than $7 \mathrm{G}$ triggers corresponding to an integrated luminosity of $70 \mathrm{pb}^{-1}$. This is probably the highest statistics ever collected in experiments with tagged photons. 


\section{FIRST RESULTS}

Data analysis searching for pentaquark is still underway. Here we report some preliminary results showing the quality of our data. Exploiting the capability of CLAS to measure simultaneously different final states we were able to check detector calibrations. Fig.1 shows some missing and invariant mass spectra for different final states detected in CLAS. Starting from the left-upper panel: $\left(\pi^{+} \pi^{-}\right)$invariant mass with a clear $K_{S}$ peak; on the right-up, $\left(n \pi^{+}\right)$invariant mass centered and the $\Sigma^{+}$peak; in the bottom-left panel $\left(n \bar{K}^{0}\right)$ invariant mass and the $\Lambda^{*}(1520)$ peak, and finally, the missing mass $M_{X}$ when a $K^{-}$is measured in CLAS with peaks corresponding to the hyperons $\Lambda(1116)$, $\Sigma(1192)$, and their excited states. These spectra were produced using only $1 \%$ of the g11 statistics!

As a check of the entire analysis procedure we derived differential (and total) cross sections for some known reactions. The luminosity was monitored and recorded during data taking while the CLAS efficiency was evaluated by mean of realistic Monte Carlo simulations. In particular, the reaction $\gamma p \rightarrow p \omega \rightarrow p \pi^{+} \pi^{-} \pi^{0}$ was studied measuring two different final states: $p \pi^{+}$and $p \pi^{+} \pi^{-}$detected. In both cases missing mass technique was used to close the kinematics. Since the two topologies mainly involve different parts of the CLAS detector a comparison of the differential cross sections extracted in the two cases is a good cross check of the entire analysis procedure. Fig. 2 shows the differential cross section measured for the two topologies and compared to the existing published data. The good agreement shows that we control the systematics errors at few percent level.

The analysis of the the reaction $\gamma p \rightarrow \bar{K}^{0} K^{+} n$ in search of evidence of the $\Theta^{+}$ pentaquark in the $n K^{+}$decay channel is currently in progress. The final channel is isolated detecting the $K^{+}$and $\bar{K}^{0} \rightarrow \pi^{+} \pi^{-}$and identifying the neutron with the missing mass technique. The direct measurement of the $K^{+}$allows one to define the strangeness of any resonance observed in this final state. Thanks to CLAS capability of detecting many particles in a wide kinematic range, the background hyperons decaying into this same final can be clearly identified and cut in the analysis. Using the full g11 data set a total number of $100 \mathrm{k} \Lambda^{*}(1520)$ were collected. When compared to the $630 \Lambda^{*}(1520)$ seen by SAPHIR, this gives a feeling of the statistical significance of g11 experiment. Results about pentaquark search will be published shortly.

\section{REFERENCES}

1. T. Nakano et al. (LEPS Collaboration), Phys. Rev. Lett. 91, 012002 (2003).

2. J. Barth et al. (SAPHIR Collaboration), Phys. Lett. B 572, 127 (2003).

3. B. Mecking et al.,Nucl. Instrum. and Meth. A503, 513 (2003). 